\title{
Del minifundio al megaincendio. Transiciones, rupturas y nuevos retos cara a un desarrollo sostenible en el medio rural gallego
}

\author{
From the small-holding to the mega-fire. Transitions, ruptures and new challenges \\ with a view to sustainable development in Galician rural areas
}

\author{
MANUEL ANGEL PAZOS LAMOSO \\ Ingeniero de Montes. Tecnologías y Servicios Agrarios, S.A., S.M.E., M.P., (Tragsatec). Grupo Tragsa \\ https://orcid.org/0000-0001-5158-9853 \\ mpazos@tragsa.es \\ XOSÉ MANUEL PALMEIRO RAMOS \\ Ingeniero de Montes. Empresa de Transformación Agraria, S.A., S.M.E., M.P., (Tragsa). Grupo Tragsa. \\ https://orcid.org/0000-0002-8584-1671 \\ xpalmeir@tragsa.es \\ DIEGO CONDE GÓMEZ \\ Doctor en Veterinaria. Tecnologías y Servicios Agrarios, S.A., S.M.E., M.P., (Tragsatec). Grupo Tragsa. \\ Grupo de Investigación de Historia Agraria e Política do Mundo Rural (HISTAGRA). USC. \\ https://orcid.org/0000-0001-9449-1397 \\ dconde@tragsa.es
}

\section{Resumen}

En un contexto de globalización, el medio rural gallego se enfrenta a nuevos retos y desafíos que no pueden ser abordados sin antes entender el origen y el alcance de estos, y que obligará a establecer un nuevo modelo de ordenación del territorio, que permita buscar soluciones a los principales problemas en un contexto social muy diferente al de épocas anteriores y con desarrollo sostenible como principal reto. En este sentido, se realiza un diagnóstico previo de las transiciones llevadas a cabo desde un modelo de aprovechamiento agropecuario mixto del medio, y que tras un profundo proceso de transformación social y del modelo productivo, supuso una especialización pecuaria y forestal, la cual a su vez convive con una mayor urbanización y dispersión sobre el territorio. A la problemática derivada de esta transformación social y productiva le debemos añadir las que se originan por unas condiciones climatológicas cada vez más extremas derivadas del proceso paulatino de cambio climático.

Dicho cambio en el modelo socioeconómico en las últimas décadas, ha derivado en un desequilibrio multifactorial y heterogéneo en diferentes áreas del medio rural, el cual consideramos es el 
desencadenante de problemáticas como el dimensionamiento y rentabilidad de explotaciones agropecuarias, el abandono y cambios de uso de tierras productivas, el aumento de las dimensiones y la peligrosidad de los incendios forestales, etc,... Para ello, se propone una ruptura de este modelo y se avanzan posibles medidas a tener en cuenta para su consecución.

Palabras clave: Galicia, sostenibilidad, ordenación rural.

\section{SUMMARY}

In a context of globalization, the Galician rural environment is facing new challenges and dares that cannot be addressed without first understanding the origin and scope of them, and that will force to establish a new paradigm of spatial planning that allows searching solutions to the main problems in a social context very different from that of previous eras and with sustainable development as the main challenge. In this sense, a preliminary diagnosis is made of the transitions carried out from a model of mixed farming use of the environment, and that after a deep process of social transformation and the productive model, involved a livestock and forestry specialization, which at its once coexists with a greater urbanization and dispersion on the territory. To the problematic derived from this social and productive transformation we must add those that originate from the increasingly extreme weather conditions derived from the gradual process of climate change.

This change in the socioeconomic model in recent decades has led to a multifactorial and heterogeneous imbalance in different areas of the rural environment, which we consider is the trigger for problems such as the increase in the danger and dimensions of forest fires, the sizing and profitability of agricultural operations, abandonment and changes in the use of productive land, etc ... For this purpose, a breakdown of this model is proposed, and possible measures are taken to consider.

Keywords: Galicia, sustainability, rural planning.

\section{INTRODUCCIÓN}

\subsection{Mirando a través del caleidoscopio del rural gallego}

La situación actual del medio rural gallego es resultado de la convergencia de diversos factores, que han ido modelando y articulando un complejo entramado que conforman un poliedro irregular, cuya forma va cambiando según los diferentes gradientes de las variables que en el intervienen, así como el punto desde el que se analice. La complejidad de dicho escenario viene determinada precisamente por esta multivariabilidad que encontramos en los diferentes espacios sociales y físicos que conforman el medio rural gallego, y el resultado de las distintas soluciones adoptadas en el pasado según los condicionantes existentes en cada uno de los procesos de transición que han ido tenido lugar. Como indicaban Grimes y Precedo Ledo (1990), este proceso tuvo efectos desiguales en cada parte de la estructura espacial del territorio gallego. Así pues, lo que inicialmente podían suponer los diferentes procesos de transición como una derivación hacia el equilibrio adaptativo en cada uno de los espacios según lo que las diferentes necesidades económico-sociales demandaban, fue convergiendo en una desambiguación entre el medio y su uso, derivando en modelos cada vez más especializados ajenos entre sí. Estos modelos 
contiguos físicamente, entran en competición no solo desde el punto de vista del espacio geográfico que ocupan, sino también desde el punto de vista de su uso socioeconómico, llegando a suponer en función del grado de separación entre ambas realidades, una ruptura de esos equilibrios históricamente establecidos.

Esta diferencia hace imposible que se pueda considerar un único factor como la causa de la ruptura de ese equilibrio. En este sentido, como veremos los diferentes planteamientos que trataban de explicar esta situación y se acercaban a desenmarañar esta circunstancia, focalizaban su interés en caras específicas del poliedro, poniendo de manifiesto la dificultad que suponían establecer soluciones que en primer lugar sirviesen para resolver dicho problema concreto, y en segundo lugar que dichas propuestas fuesen posible ponerlas en marcha de una manera homogénea en la totalidad del medio rural gallego.

En la misma línea que exponía López Iglesias (2000), este carácter poliédrico, obliga a diseñar y desarrollar medidas concretas en función de las diferentes características que presenta la heterogeneidad de dicho medio.

\subsection{Un análisis de la evolución del minifundio al megaincendio}

Partiendo de la premisa que no hay explicaciones simples para problemas complejos, en este trabajo se pretende explicar el proceso que nos ha traído a esta situación y orientar de forma preliminar desde un punto de vista propositivo, los diferentes itinerarios desde los cuales se pueda revertir y abordar los principales desafíos a los que se enfrenta el medio rural gallego.

En este sentido, se realiza una revisión previa de las diferentes transformaciones que se han producido recientemente en el sistema agropecuario y forestal, así coma la valoración de las principales problemáticas que dichos cambios han provocado en la gestión de dicho medio. Así mismo se describen las tendencias futuras y los nuevos retos a los que se enfrentará el medio rural gallego y al que deberemos hacer frente a través de una sociedad muy diferente a la existente hace apenas unas décadas. No se trata pues de llevar a cabo un análisis cuantitativo del dicho proceso en los diferentes escenarios que lo conforman, cuestión que como veremos ya ha sido abordada. Consideramos que más allá de la valoración analítica pormenorizada de la evolución de diferentes condicionantes, es necesaria hacer una evaluación global de la problemática en donde se tenga en cuenta el proceso dinámico que ha vivido el medio rural, a través de un esquema definido por una situación inicial, una readaptación/transición condicionada y la ruptura del equilibrio preestablecido. De alguna manera, se pretende explicar cómo el paso desde una agricultura minifundista pueda dar lugar a problemáticas actuales como los megaincendios, que no son más que la manifestación de esas rupturas subyacentes.

Para ello, se parte de una primera revisión de la conversión del medio rural gallego, a través de las diferentes transformaciones que a nivel productivo han condicionado los cambios en el sistema agropecuario gallego y el balance medioambiental que dicha evolución supuso, teniendo en cuenta las transformaciones territoriales, sociales y productivas 
que han sucedido en Galicia. En este sentido, a continuación, describimos la situación actual como rotura del equilibrio existente, y los efectos que esta inestabilidad provocan en el conjunto del complejo escenario que indicábamos al inicio. Teniendo en cuenta este entramado dinámico, donde persisten multitud de variables, se pretende comenzar a diseñar herramientas que permitan afrontar de una manera sostenible el desarrollo equilibrado del medio rural gallego, de tal forma que nos orienten las líneas de trabajo futuras que deberán ser definidas y desarrolladas en otros trabajos posteriores al actual.

\section{RUPTURAS DEL EQUILIBRIO EN EL SISTEMA AGROPECUARIO GALLEGO}

\subsection{De la rubia a la pinta. Transición cara la ganadería intensiva}

El proceso de frisonización del campo gallego desde mediados del siglo XX supuso un desplazamiento en el censo pecuario de razas autóctonas que hasta ese momento tenían un carácter productivo de triple aptitud (carne-leche-trabajo) (Martínez López, 2000). La incorporación de nuevas razas especializadas en la aptitud lechera, así como la mecanización del campo, derivó que estas razas autóctonas se dirigieran cara la producción cárnica en el mejor de los casos, o cara una situación de marginalidad en el caso de razas más rusticas (Conde Gómez, 2014). Trabajos recientes de Lanero Táboas (2016) y Otero Rodríguez (2018a, 2018b), abordan este proceso de frisonización y ponen de manifiesto como en un contexto de aplicación de revolución verde, supone un proceso de ruptura respecto a los modelos productivos anteriores basado en el mixed farming y enfocados a un aprovechamiento mixto del ganado. Dicho proceso no es exclusivo de la ganadería vacuna gallega. El desarrollo de otros tipos de ganadería intensivas como la avicultura o la porcinocultura, especialmente concentrado en la provincia de Ourense (Fernández Martínez, 1975), acentúan la cada vez mayor disociación entre ganadería y territorio. Este modelo de ganadería industrial, está unido a un proceso de transformación en el modelo de la alimentación animal, con una fuerte dependencia de la importación de cereal para la elaboración de pienso, detrimento del uso de forrajes producidos en la proximidad de la explotación (Soto Fernández et al., 2016). Sin embargo, este modelo plantea importantes dificultades a la hora de mantener la estabilidad de las explotaciones ganaderas. Dicha especialización, tiene una clara dependencia de la volatibilidad del mercado, bien para la adquisición de inputs, comopara la comercialización de sus producciones. El abaratamiento de costes que permitan la viabilidad de las explotaciones, así como los requerimientos agroambientales exigidos por la Política Agraria Común, llevan necesariamente a que las explotaciones tengan que dimensionar su base territorial, lo cual en la actualidad choca indefectiblemente con el resto de los aprovechamientos, así como la incapacidad de movilización de tierras abandonadas.

En la situación actual, donde se está produciendo una nueva reconversión del sector lácteo gallego, es fundamental revisar el potencial productivo de las razas autóctonas, los 
cuales fueron abandonados por el proceso de especialización lechera, recuperando líneas de mejora que supongan una alternativa productiva en un contexto de mayores exigencias medioambientales y de rentabilidad de las explotaciones ganaderas.

\subsection{Entre pinos y eucaliptos. La transformación forestal}

La transformación forestal que se llevó a cabo en Galicia a lo largo del siglo XX fue muy profunda. Se puede afirmar que a comienzos del siglo XX contábamos con un sistema forestal complementario al ganadero y al agrario, cuya finalidad, a parte de la obtener leña para el cocinado y la calefacción doméstica o madera para carpintería o construcción, también era la de proporcionar nutrientes para fertilizar las tierras de labor, material vegetal para las camas del ganado o alimento para la ganadería extensiva, y hemos terminado el siglo XX con un monte con una finalidad básicamente productora de madera para procesos industriales (tablero, pasta de papel y aserrado) y con escasa conectividad con el sector agrícola y ganadero. Se produjo una evidente desconexión física y de flujos de productos entre lo agrícola y ganadero y lo forestal.

Entre finales del S.XIX y la primera mitad del S.XX se produce en Galicia una importante y constante acción repobladora con la introducción principalmente del pino marítimo (Pinus pinaster Ait.) debido a la conjunción de la crisis finisecular caracterizada por el descenso de precios agrícolas, con un aumento de la demanda de productos de aserrío del pino (tabla y tablillas para envases y apeas para minas) que supuso un incremento de su precio. Este hecho, que convirtió la repoblación con pino en una buena opción a corto-medio plazo, posibilitó la apertura de un importante número de aserraderos, hasta llegar a los 1.000 a finales de 1940 (Rico Boquete, 2014).

Desde mediados del S.XX hasta el día de hoy el sector forestal ha seguido transformándose en una adaptación continua de la industria al mercado, y de los montes y la selvicultura a la industria. Esa transformación se puede resumir en una especialización todavía más acusada de la superficie forestal como productora de madera, sin apenas otros aprovechamientos secundarios o complementarios y en la fuerte expansión del eucalipto (Eucalyptus globulus Labill, principalmente) en nuestros montes.

Si bien esta especie lleva con nosotros desde el 1850 (Silva-Pando and Pino-Pérez, 2016), es a partir de los primeros setenta y posteriormente en los 90 cuando se confía al eucalipto la rentabilidad económica de las propiedades forestales de los gallegos (Marey Pérez et al., 2017), un proceso que los sucesivos inventarios forestales (IFN1 1973 a IFN4 2009) confirman, incrementándose en más de un 300\% la superficie de eucalipto (masas puras y mezclas con otras especies), siendo significativo el impactante incremento de las masas puras pasando de 27.418 ha según el IFN1 de 1973 a 287.983 ha en el IFN4 de 2009.

¿Qué nos ha llevado a esta fuerte expansión? pues el conjunto de actividades realizadas libremente por los agentes económicos sin intervención del poder público, es decir, el mercado. Un mercado muy diferente al que impulsó la implantación del pino entre 
finales del s. XIX y primera mitad del s. XX, pero en el que sigue operando las mismas reglas de oferta y demanda de productos y de la rentabilidad económica como criterio de elección de especie.

Ha sido la fabricación de un nuevo producto en Galicia, la pasta de papel, el que ha cambiado las reglas existentes. En 1963 entró en funcionamiento en Pontevedra la Empresa Nacional de Celulosa, con la pretensión inicial de consumir principalmente pino en su proceso productivo para la fabricación de 30.000 t/anuales de pasta de papel. Sin embargo esta industria ha ido modificando su sistema productivo de tal forma que en la actualidad produce del orden de 465.000 t/año de pasta de papel a partir de eucalipto, principalmente E. globulus, debido a las ventajas que esta especie proporciona por la morfología de su fibra y la composición química de su madera (Bermúdez Alvite et al., 2002). Un proceso similar sucedió en 1973 con la puesta en funcionamiento de la empresa pastera CEASA (Celulosas de Asturias S.A.) en Navia, que también tiene incidencia por su demanda de madera gallega y que en la actualidad produce más de 600.000 t/año de pasta de eucalipto.

Por tanto, podemos ver como la adaptación entre el monte y la industria se ha producido en una doble dirección, primero la industria se adaptó al medio demandando sus recursos y posteriormente ha sido el medio el que ofrece sus recursos adaptándolos a la demanda de la industria.

En los últimos años, el eucalipto ha centrado buena parte del debate siendo visto por muchos ciudadanos como el causante de buena parte de la problemática incendiaria actual y de la degradación del medio forestal. Sin embargo los trabajos que han abordado e incorporado esta problemática desde el punto de vista de la crítica social, económica y ambiental son escasos (Soto Caba, 2013). Uno de estos escasos trabajos que abordan la globalidad de esta cuestión es el informe de Greenpeace (Veiras and Soto, 2011), en el cual indica que "el problema no es el eucalipto, son las políticas públicas (o la ausencia de políticas)". En la misma línea otros autores consideran que el problema del eucalipto es complejo y poliédrico pasando de ser una discusión técnica, socioeconómica o ambiental a convertirse en una discrepancia social con bandos bien definidos. Para estos autores "el problema actualmente existente en torno al género Eucalyptus spp. en Galicia no debe fundamentarse en la "buena o mala fama", si no si se está realizando una gestión forestal adecuada en base a una planificación del territorio forestal" (Marey Pérez et al., 2017).

Una mayor diversidad espacial y discontinuidad de los tipos de combustible dificulta la propagación del fuego y favorece el ataque, haciendo que el incendio sea menor (Loepfe et al., 2011; Viedma et al., 2015). En el caso de Galicia comprobamos que el incremento en la extensión de la masa forestal y también de sus existencias por hectárea que refleja el $4^{\mathrm{a}}$ Inventario Forestal Nacional, supone una mayor propagación del fuego y una mayor dificultad en la extinción del incendio pudiendo llegar a obtener intensidades tan elevadas que por más recursos de extinción que se dediquen no se podrá influir en el comportamiento del fuego. 


\subsection{El mercado como condicionante de la ruptura}

En todo este proceso, la especialización de las producciones agropecuarias y forestales gallegas han ido cara a una economía de mercado globalizado donde el factor determinante es la competitividad en el precio final del producto, ha supuesto una evolución hacia la producción industrial de bienes de escaso valor añadido. En el ámbito agropecuario, las producciones agrícolas y ganaderas se han ido especializando hacia sistemas cada vez más dimensionados, automatizados y aislados del medio sobre el que se asienta y donde la competitividad en el precio del producto final es prácticamente el único elemento que permite la subsistencia de estas explotaciones. En ámbito forestal se ha basado en la fabricación y producción de mobiliario empleando madera de trituración (tablero aglomerado, de fibras, rechapados...) sin ningún tipo de intervención artesanal ni de diseño a medida, donde la obtención de un producto estandarizado a bajo precio se convierte en el mayor reclamo del producto.

No se trata de una tendencia aislada ni exclusiva del sistema agropecuario o forestal, sino que es una tendencia social en el que el low-cost se ha impuesto en buena parte de la sociedad, y que se observa también en la producción de ropa y complementos, de productos tecnológicos, electrodomésticos... Sin embargo, sí tiene una característica propia, y es su capacidad para incidir en el medio y el territorio, cuestión esta que no se produce en otros sectores.

En Galicia somos en general productores de bienes de escasa transformación y valor añadido. De esta forma somos uno de los principales productores de leche líquida pero no de producción de yogures, quesos o leche en polvo. Producimos enormes cantidades de tablero, pero no disponemos de una industria de la carpintería y mobiliario acorde con esa capacidad. Somos uno de los principales productores de pasta de papel pero no de producción de productos celulósicos derivados (papel de escritura, papel fotográfico, papel tisú,...). Somos un gran productor de productos de la minería como pizarra o granito, pero buena parte de ellos en bruto y sin apenas procesado.

La producción low-cost también trae consigo materia prima y salarios low-cost. Esa materia prima que se emplea para fabricar esos productos carece apenas de valor y por tanto de rentabilidad en un territorio como Galicia donde la falta de una ordenación territorial dificulta la economía productiva de escala, que es la única que podría proporcionar productos a bajo precio y mantener igualmente la rentabilidad de las explotaciones.

Es por tanto indispensable romper con esa tendencia dañina y empobrecedora para que nuestros productos puedan ser generadores de procesados industriales posteriores, y que no sea cuestión exclusivamente de producir más cantidad y a menor precio, si no también de fabricar productos de mayor valor añadido. Sectores como el vitivinícola o el conservero han sido capaces de demostrar que es posible realizar este modelo también en Galicia. Debemos ser capaces como sociedad de apostar por productos que reflejen los valores medioambientales o éticos a los que aspiramos. 


\subsection{La interfaz urbano-agroforestal otro reflejo del desequilibrio}

En Galicia todo es transición (Armas Diéguez, 1997). Transición en el poblamiento "inmueble" son los hábitats tradicionales dispersos que existían (y existen) entre la concentración absoluta de las ciudades y la diseminación absoluta de los caseríos; hábitats en los que el tamaño de las aldeas dependía de la superficie agrícola útil y de la fertilidad de los suelos (Niemeier, 1945).

Transición en el poblamiento semoviente fue el movimiento de población del campo a la ciudad que, de una forma acelerada, comienza en el rural gallego en los 70, acrecentándose de tal manera entre los mediados 80 y principios del nuevo siglo, que en el 2005 ya se podía afirmar que Galicia era ya una sociedad urbana (Precedo Ledo et al., 2008). Este proceso de desruralización que en Galicia ocurre de modo retrasado con relación a otros territorios españoles ha sido muy intenso, en especial en las últimas décadas del siglo XX (Vázquez González et al., 2008).

Así, la sociedad rural eminentemente agraria de principios/mediados del siglo pasado en la que su hábitat disperso dibujaba un mosaico racional de usos del suelo en el que los agricultores eran depositarios de amplias experiencias sobre los modos de manejo integral del medio natural en el que se desenvolvían (Gallego Martínez, 2014) se convierte en pocas décadas en una zona decaída que deriva en un progresivo abandono de núcleos y en una pérdida del mosaico racional de usos existentes hasta la fecha que provoca un acercamiento del "monte" a las viviendas.

Así mismo, nos encontramos en la actualidad ante un nuevo proceso de desconcentración urbana que va paralelo al incremento del nivel de vida y a la masificación del automóvil entre la población, teniendo un impacto territorial que se refleja en un aumento de la diseminación y una mayor heterogeneidad morfológica del hábitat, puesto que se sobreimpone al medio rural (Armas Diéguez, 1997).

Este proceso lleva también asociada una nueva estética de lo rural que los nuevos pobladores tienen en su cabeza, alejada de la del agricultor que, no ve lo bonito o lo feo, solo cree en lo que es susceptible de producir algo o ser una amenaza para la producción.

Este proceso de desconcentración urbana se ha superpuesto sobre unos asentamientos ya completamente diseminados de antemano, enquistando en el paisaje una presencia mixta y entrelazada de horizontes industriales, urbanos o rurales en franjas muy extensas y alejadas varios kilómetros de los centros de las metrópolis gallegas, originando una nueva trama edificada que ha superado ampliamente los límites administrativos de los municipios y ha originado la creación de una verdadera y original ciudad difusa. Este nuevo desarrollo es un gran consumidor de suelo, energía y recursos naturales, simbolizando paradójicamente una forma de desarrollo no sostenible, que soporta unos costes de infraestructuras y de gestión excesivos (Otero-Enríquez and Gómez Rodríguez, 2007), a lo que se suma, sobre todo en las zonas de clima mediterráneo, un incremento del riesgo de incendios (Badia et al., 2010) 
Pues bien, esta nueva transición en el territorio gallego nos lleva a un proceso inverso pero perversamente complementario al acaecido en la primera transición, un acercamiento de la urbanización al monte.

Se ha producido en consecuencia una disociación del modo de habitar; el propietario de tierras agroforestales, el residente en el territorio y el que genera actividad agraria se separan, en el mismo espacio donde antes estos tres factores formaban un único sistema coherente. Y esa disociación provoca que buena parte de las medidas y políticas agrarias no tengan repercusión real sobre el territorio puesto que ni los propietarios de la tierra ni sus habitantes ejercen actividad agraria alguna.

En este espacio dominado por una urbanización dispersa, Stewart et al. (2007) lo denominó wildland-urban interface o interfaz urbano-forestal (UIF). Esta interfaz urbano-rural tenía en el 2012 una extensión de 244.187 hectáreas, lo que representa el 8,3\% de Galicia, siendo más abundante en zonas específicas de la geografía gallega como en el sur-oeste de Galicia (Chas Amil, 2013).

Así pues, las transiciones campo-ciudad/ciudad-campo han convertido a Galicia en el paradigma de la maximización del riesgo de incendios, su territorio experimenta una progresiva expansión de la interfaz urbano-forestal que, asociada a la dispersión del sistema de asentamientos y a la progresión de la vegetación natural, constituye una dinámica territorial preocupante en relación con el problema de los incendios forestales (Galiana Martín, 2012).

De esta forma aparece una nueva tipología de incendios que se propaga sobre un nuevo combustible: las urbanizaciones. Se trata de la $5^{\text {a }}$ generación de incendios (Padrón Castañeda and Barranco Reyes, 2014) y en ella, ya no tenemos un fuego que quema masa forestal y puede afectar a la vivienda, sino un incendio que se propaga por masa forestal y jardines o casas sin dificultad ni diferenciaciones.

\subsection{El cambio climático, un nuevo punto generador de rupturas}

La existencia del cambio climático está fuera de cualquier duda técnica o científica y según el Panel Intergubernamental de Expertos sobre Cambio Climático (IPCC), "es extremadamente probable -más del 95\%-que la influencia humana sea la causa dominante del calentamiento observado desde mediados del siglo $X X$ ', si bien sí puede ser objeto de discusión su intensidad y consecuencias. En el Quinto Informe de Evaluación del IPCC (Grupo Intergubernamental sobre Cambio Climático) de 2014 se señala un claro riesgo de daños a causa de fenómenos extremos relacionados con el calor e incendios forestales en el medio y largo plazo con incrementos de temperaturas entre $2^{\circ}$ y $4^{\circ}$, categorizando este riesgo como alto o muy alto con el nivel de adaptación actual y rebajándolo a medio/alto presuponiendo que el potencial de adaptación que presupone que existe se aprovecha y se toman medidas para minimizar este riesgo.

Así en vista de los datos de este y de otros estudios que muestran un aumento sostenido de los HTDs (días de altas temperaturas) en el arco mediterráneo, hipotéticamente 
asociado al incremento generalizado de las temperaturas resultante del cambio climático, y empíricamente vinculado al desarrollo de grandes incendios forestales, (este último dato es tan relevante que se ha estimado que un elevadísimo porcentaje de la superficie quemada al año en España se produce durante estos días, que representan un creciente pero aún pequeño porcentaje de los días del año) (Padrón Castañeda and Barranco Reyes, 2014), habrá que plantearse como un hecho consumado que el cambio climático está aquí para quedarse como un actor principal en el desarrollo de los megaincendios en Galicia.

Las diferentes proyecciones y modelos climáticos que se realizan coinciden en que habrá una mayor severidad del tiempo atmosférico para los incendios en el futuro, aumentando la ocurrencia y la severidad de los mismo, lo cual plantea serias dudas sobre la capacidad de los sistemas de extinción para su control (Flannigan et al., 2009).

\section{EL NECESARIO CAMBIO DE MODELO}

\subsection{Cara donde avanza el medio rural del siglo XXI}

Hemos visto como la rotura de los equilibrios preexistentes provoca tensiones entre los diferentes actores que conforman las nuevas realidades del espacio rural. El cambio profundo de modelo social y territorial que se ha expuesto hasta aquí ha ido sucediendo poco a poco, de forma poco perceptible y mediante una sucesión de pequeños y múltiples cambios en el territorio en la misma dirección durante las últimas década y en la actualidad sus efectos son evidentes: pérdida de población en el ámbito rural, importantísimo descenso del empleo agrario, la desestructuración territorial en el rural (López Iglesias, 2018), el abandono de tierras agrarias (Corbelle Rico and Crecente Maseda, 2008), disminución de la superficie agraria útil que ha supuesto un aumento de la superficie forestal y la especialización productiva, bien mediante la colonización de matorral y frondosas caducifolias o bien mediante la plantación de cultivos forestales intensivos (Eucalyptus spp en su mayoría) (Soto Fernández, 2016).

Todos estos cambios en el ámbito agrícola, ganadero y forestal, tienen consecuencias medioambientales como el aumento del riesgo de megaincendios como han demostrado hasta la fecha múltiples autores (Barreal Pernas et al., 2012, 2011; Vicente Serrano et al., 2000) o la problemática de un descenso de biodiversidad en estos medios (Tello, 2015; Tello et al., 2018). Todo ello son diferentes caras de un mismo problema y las consecuencias de estos cambios indican la incompatibilidad e insostenibilidad de este modelo productivo. Esto obliga a evaluar y analizar las amenazas y oportunidades de los nuevos tipos de aprovechamiento agrícola, ganadero y forestal para las próximas décadas, de manera que nos hagan comprender cómo podrán afectar al desarrollo rural gallego en el futuro.

El sector agropecuario participa de los grandes desafíos a los que debe dar respuesta la humanidad en las próximas décadas. Si damos por válida la hipótesis más aceptada de las Naciones Unidas en la que se estima que habitaremos este planeta 9.700 millones 
de personas en el año 2050, será necesario incrementar un 1,8\% anual la capacidad para producir alimentos y hacerlo consumiendo menos recursos naturales.

Por otro lado, la conciencia ambiental y de alimentos saludables de nuestras sociedades es cada vez mayor, la demanda de productos procedentes de la agricultura ecológica, iniciativas sociales como SlowFood o de reducción del desperdicio de alimentos es la prueba de esa nueva conciencia social.

La agricultura por tanto no es ajena a ambas tendencias y se encamina hacia dos modelos en principio contradictorios pero llamados a convivir como son la agricultura de precisión y la agricultura de alto valor natural.

La agricultura de precisión es un concepto moderno de gestión agropecuaria que utiliza técnicas digitales (GPS, sensores, imágenes satélites...) para hacer un seguimiento de los procesos de producción agraria buscando su máxima optimización (Schrijver et al., 2017). Su objetivo es simple, ahorrar costes de producción para producir más alimentos con menor consumo de materias primas (agua, energía, abonos, plaguicidas...).

Se trata de una agricultura altamente tecnificada y mecanizada, capaz de producir mayores cantidades de producto con menos consumos de materia prima y por tanto capaz de producir un producto agrario a bajo coste. Para ello generalmente precisa de grandes extensiones agrarias que cultivar para así rentabilizar las elevadas inversiones necesarias, de agricultores altamente formados y de cultivos genéticamente mejorados. Es altamente competitiva con reglas de mercado y necesita menos apoyo público que otras formas de producción agraria. Sin embargo, esta agricultura también tiene sus contras, y de esta forma implicará una reducción de la mano de obra necesaria para la obtención de productos agrarios, que los productos obtenidos tengan un coste muy bajo que hará muy difícil competir a la agricultura tradicional, que se produzca una pérdida de variedad genética y de biodiversidad o que será una agricultura poco accesible debido a los elevados costes de inversión y la alta especialización necesaria.

En contraposición con este modelo cada día aumenta la conciencia medioambiental de nuestras sociedades y también de los estándares de alimentación. Las normativas y los hábitos en materia de residuos, energía, alimentación, etc. buscan ser más ecológicos y eficientes, evitando el actual nivel de empleo de los recursos naturales. Así mismo la sociedad está cada vez más concienciada sobre su alimentación y el origen de sus alimentos, con productos bio, ecológicos, de temporada, de cercanía y más saludables.

De esta forma la agricultura ecológica en la UE se ha desarrollado rápidamente durante los últimos años. Según los datos de Eurostat, la UE-28 tuvo en 2015 una superficie total de 11,1 millones de hectáreas cultivado como orgánico, frente a los de 5.0 millones en 2002. Durante la última década, el área orgánica en la UE aumentó en aproximadamente 500.000 hectáreas por año, representando del orden del 6,2\% del área agrícola total utilizada en Europa (DG Agriculture and Rural Development, 2016).

Existen aproximadamente 185.000 granjas ecológicas en toda Europa. La mayoría de la superficie ecológica (78\%) y de las granjas ecológicas (81\%) están situadas en los Estados miembros de la UE que se unieron a la UE antes de 2004 (UE-15), en la que su 
legislación, entre otros, ayudó a estimular el desarrollo de este sector. Los países que se han unido a la UE desde 2004 tampoco se han quedado rezagados registrando incrementos del 12\% anual en su superficie de producción ecológica en el periodo 2002 a 2015 y aumentaron el número de explotaciones casi diez veces entre 2003 y 2015.

En este sentido cada vez se habla más de la agricultura de Alto Valor Natural (High Nature Value -HNV- en inglés) como aquellos sistemas de producción agraria caracterizados por su larga implantación en el territorio, por su baja intensidad en el consumo de recursos y a menudo compleja, que emplea recursos intensivos de mano de obra, así como razas de ganado y cultivos altamente adaptados a los suelos locales, la vegetación y el clima.

Este tipo de aprovechamiento ha transformado y mantenido hábitats de gran importancia para la biodiversidad y su conservación es de especial importancia a través de las medidas de conservación de la UE. Estas directivas persiguen que los estados miembros adopten medidas para conservar hábitats y especies amenazadas, de los cuales 57 hábitats y 257 especies dependen o están asociadas con actividades agrarias típicas de este sistema de aprovechamiento tradicional.

Se trata sin embargo de una agricultura y una ganadería económicamente vulnerable porque muchas veces sus productos compiten con los procedentes de otros sistemas productivos mucho más intensivos capaces de ofrecer productos similares a mucho menor precio. Además, los tienen que producir en zonas poco pobladas, donde sus habitantes tienen escasos servicios lo que dificulta su asentamiento.

La baja productividad de estos sistemas y su elevada demanda de mano de obra en comparación con la agricultura y ganadería intensiva aboca a este tipo de explotaciones a una desventaja competitiva que sólo se está resolviendo parcialmente con ayudas de la PAC o de los programas de desarrollo rural, que muchas veces no diferencia lo suficiente entre los diferentes sistemas productivos.

\subsection{Galicia ante las tendencias globales}

En el caso de Galicia ambos tipos de sistemas de producción agroganadero no se darían por igual en todo el territorio. Siguiendo la caracterización que Corbelle Rico y Crecente Maseda (2014) hacen de Galicia según su tipología entre rural urbanizado y forestado, rural activo y rural abandonado (rural abandonado-frondosas / rural abandonadomatorral), se podrían observar grandes diferencias en materia demográfica, de superficie media de las parcelas, tipología de cultivos, grado de mecanización, acceso a servicios, valores naturales a proteger, etc.

Con estas premisas la agricultura de precisión que hemos comentado parece encajar especialmente en aquellas parroquias definidas como rural activo y que podemos asimilar a la Galicia interior, donde se podría esperar una alta especialización agraria, un continuo aumento de la superficie media por explotación, una mayor mecanización y tecnificación de las labores agropecuarias. 
Por la contra, la agricultura de alto valor natural tiene especial acomodo en las parroquias definidas como Rural abandonado y que se corresponde con la Galicia de montaña. En estas parroquias destaca la importancia de sus valores naturales, paisajísticos y de conservación de la biodiversidad, unido con una menor capacidad productiva derivadas de su clima y de su suelo, su baja demografía y escasez de servicios hacen que las políticas públicas que fomenten la agricultura de alto valor natural tengan especial impacto e importancia.

En aquellas parroquias de la Galicia atlántica que se podrían corresponder con el denominado de rural urbanizado y forestado, el papel de las producciones agrarias y su importancia en términos económicos y de ocupación del territorio es mucho más pequeña que en otras zonas. Se ha constatado que en estas áreas con población más joven y demográficamente estable desaparece la superficie agrícola periurbana, desplazada por la urbanización y la forestación (Corbelle Rico and Crecente Maseda, 2014). Se suele producir, y cada vez con menos intensidad, una agricultura de autoconsumo con pequeñas huertas próximas a las viviendas, pudiendo existir alguna explotación ganadera aislada pero generalmente de escasa dimensión. En estas parroquias parece que el principal valor de la tierra es el que pueda derivar del urbanismo en base a su expectativa de poder ser edificada. Por todo ello las políticas en materia agraria apenas podrán tener incidencia en estas áreas, siendo en estos casos necesario establecer medidas dirigidas a establecer un mayor control urbanístico en las que se establezcan espacios de usos diferenciados y de zonas de especial seguridad, de manera que limiten el riesgo que esta interfaz tiene al respecto de cuestiones como los incendios forestales.

\section{PROPUESTA ANTE UN CAMBIO DE PARADIGMA}

\subsection{Intensificación y discontinuidad}

Hemos visto como en las últimas décadas se ha producido una intensificación y especialización de las producciones, tanto las forestales como las agrícolas y ganaderas, produciéndose una rotura casi total entre las conexiones de estos sectores, históricamente ligados de forma íntima y difícilmente separable. Ni el ganadero necesita tierra agraria útil para alimentar a sus animales y gestionar sus purines, ni el agricultor necesita estiércol que produce la ganadería o material vegetal de los montes, ni los silvicultores emplean al ganado para controlar la vegetación de sus plantaciones.

Mantener la homogeneidad y estanqueidad de los espacios agrarios, en función de la determinación física, social o económica del territorio, no hace más que agravar esa ruptura de nexos, que desestabilizan dicho equilibrio. Políticas caducas basadas en la promoción de la propiedad como medio de regeneración del territorio bien a través de las iniciativas de concentración parcelaria o a través del subsidio de la construcción y renovación de viviendas, no han servido para solucionar problemas como la falta de base territorial o el abandono de la superficie agraria (Gkartzios and Norris, 2011). 
Así, la heterogeneidad de los diferentes modelos adoptados en los espacios y sistemas agropecuarios, exigen que su interpretación sea abordada desde diferentes enfoques interconectados a modo de red, donde la prioridad del uso adecuado del territorio tiene que primar por encima de la propiedad. Dentro de ese cambio de paradigma, establecemos la necesidad de conformar una red (Cooke and Morgan, 1993; Esparcia, 2014; Murdoch, 2000) que permita combinar las dos tendencias agropecuarias actuales (agricultura y ganadería de precisión y la agricultura de Alto Valor Natural) en un mismo espacio, capaz de movilizar el uso del territorio de una manera dinámica. Estos espacios heterogéneos caracterizados por un conjunto de usos de la tierra que poseen alteraciones contrastantes proporcionan una contribución sinérgica a la conservación de la biodiversidad (Marull et al., 2018).

Así, las iniciativas públicas dirigidas al desarrollo rural deben favorecer la creación de nuevas correlaciones entre los diferentes nodos ya establecidos, creando nexos de unión donde se complementen ambos tipos de explotaciones agropecuarias, en los que se implantarían modelos de explotación mixta, en la que de manera simbiótica cada uno de los espacios fuese creciendo.

Poner en marcha este tipo de medidas encaminadas hacia este tipo de modelos de aprovechamientos mixto, exige en primer lugar que desde la administración se aborden primero una catalogación y ordenación del territorio basado en el uso que se hace del mismo, de manera que se puedan precisar los limites existentes en cada uno de los espacios y definir los nodos y nexos primarios, así como los índices de sostenibilidad ambiental (ISA) para cada uno de los espacios e interacciones definidas. Una vez se establezcan un patrón tipo, dependiendo de las condiciones iniciales, se deberán adoptar medidas específicas que permitan la movilidad del uso del territorio, de tal manera que los nuevos aprovechamientos tengan en cuenta un incremento en el ISA de cada nodo. En esta línea, ya existen trabajos previos que pueden servir de base (Borobio Sanchiz et al., 2015; Pérez-Alberti et al., 2014), pero que sin embargo deberían incluir necesariamente una utilidad de aplicación práctica en el territorio.

\section{CONCLUSIONES PARCIALES}

La problemática actual del medio rural está ligada a la rotura de los tradicionales equilibrios y aprovechamientos del agro gallego agravado por un escenario de desorden urbanístico y cambio climático que provoca incendio de mayor magnitud y de mayor peligrosidad para la población. Una problemática que por otra parte es compartida en otros lugares aparte de Galicia como pueden ser otras regiones de España, Portugal o Grecia.

De los tres principales factores generadores es preciso que a corto plazo se actúe sobre dos de ellos; el sistema agrario y el urbanismo, deben ser objeto de un debate y de un replanteamiento de estrategias a corto plazo. Esta situación tiene por tanto mucho que ver con el fenómeno de desestructuración agraria que se ha producido en las últimas décadas. Esta ruptura, agravada por problemas de carácter social como el abandono de la 
actividad agraria, envejecimiento del rural o el incremento de las interfaces con el medio urbano; problemas económicos como la concentración del capital y desambiguación de los beneficios ajenos a una reinversión de la plusvalía en el espacio donde se producen; ha supuesto un elemento fundamental para la existencia de los megaincendios,

Ante esta nueva situación es por tanto imprescindible intensificar la ordenación sobre el medio mediante la redistribución y adecuación de los usos del territorio, así como fortalecer los vínculos e interrelaciones entre los diferentes nodos y modelos productivos que favorezcan aprovechamiento mixto del territorio, de manera que manteniendo discontinuidades sobre el terreno se garanticen usos compatibles. En este sentido se deberían abordar nuevos debates cara el establecimiento de medidas encaminadas hacia una economía de densidad sostenible, en las cuales se establezcan medidas encauzadas a restaurar ese equilibrio. De esta manera, este trabajo es preludio de dos líneas de trabajo práctico dirigidas en un primer lugar en dar un corpus legislativo que sirva de base para permitir los cambios necesarios para un uso equilibrado del territorio, así como permitir la instauración de nuevos nexos que favorezcan un desarrollo homogéneo de la actividad agropecuaria y forestal (movilización de la propiedad, autoabastecimiento alimentario en granjas, gestión de biomasa, etc). En segundo lugar, este proceso debe pasar por la categorización de los usos actuales del territorio, de manera que se pueda establecer cuáles son los diferentes componentes principales que permiten un uso equilibrado y sostenible del territorio, de manera que en aquellas zonas donde exista variación al respecto del modelo ideal, se puedan facilitar patrones con los que revertir esta variación. 


\section{BIBLIOGRAFÍA}

Armas Diéguez, P., 1997. Aldea y paisaje, patria y patrimonio, in: O Camiño inglés e as rutas atlánticas de peregrinación a Compostela. II Aulas no Camiño. Universidade da Coruña, Ferrol, pp. 105-139.

Badia, A., Estany, G., Otero, I., Boada, M., 2010. Una aproximación al estudio del crecimiento urbano disperso en Catalunya (1956-2008) y la aportación de las fuentes orales a la interpretación de los cambios en el paisaje. Boletín de la Asociación de Geógrafos Españoles, 2010.

Barreal Pernas, J., Loureiro García, M.L., Picos Martín, J., 2012. Estudio de la casualidad de los incendios forestales en Galicia. Economía agraria y recursos naturales 12, 99-114.

Barreal Pernas, J., Loureiro García, M.L., Picos Martín, J., 2011. Estudo da incidencia dos incendios en Galicia: unha perspectiva socioeconómica. Revista galega de economía: Publicación Interdisciplinar da Facultade de Ciencias Económicas e Empresariais 20, 227-246.

Bermúdez Alvite, J., Touza Vázquez, M., Sanz Infante, F., 2002. Manual de la madera de eucalipto blanco. Fundación para o Fomento da Calidade Industrial e Desenvolvemento Tecnológico de Galicia, Ourense.

Borobio Sanchiz, M., Turrado Sánchez, J.D., Pérez Alberti, A., 2015. Fuentes oficiales de información territorial y su integración para la cartografía de los agropaisajes gallegos. Semata: Ciencias sociais e humanidades 39-67.

Chas Amil, M.L., 2013. Delimitación de la Interfaz Urbano-Forestal en Galicia: Análisis del riesgo de incendio. Congreso Forestal Español.

Conde Gómez, D., 2014. Canto val unha vaca? Da cuestión agraria á cuestión pecuaria. Deputación Provincial da Coruña, A Coruña.

Cooke, P., Morgan, K., 1993. The network paradigm: new departures in corporate and regional development. Environment \& Planning D: Society \& Space 11, 543-564. https://doi.org/10.1068/d110543

Corbelle Rico, E., Crecente Maseda, R., 2014. Urbanización, Forestación e abandono. Cambios recentes na paisaxe de Galicia, 1985-2005. Revista galega de economía: Publicación Interdisciplinar da Facultade de Ciencias Económicas e Empresariais 23, 35-52.

Corbelle Rico, E., Crecente Maseda, R., 2008. El abandono de tierras: concepto teórico y consecuencias. Revista Galega de Economía 17, 0.

DG Agriculture and Rural Development, 2016. Facts and figures on organic agriculture in the European Union. Comisión Europea.

Esparcia, J., 2014. Innovation and networks in rural areas. An analysis from European innovative projects. Journal of Rural Studies 34, 1-14. https://doi.org/10.1016/j. jrurstud.2013.12.004

Fernández Martínez, G., 1975. Galicia y las Cooperativas Orensanas. Dagur, Madrid. 
Flannigan, M.D., Krawchuk, M.A., de Groot, W.J., Wotton, B.M., Gowman, L.M., 2009. Implications of changing climate for global wildland fire. International Journal of Wildland Fire 18, 483. https://doi.org/10.1071/WF08187

Galiana Martín, L., 2012. Las interfaces urbano-forestales: un nuevo territorio de riesgo en España. Boletín de la Asociación de Geógrafos Españoles 58, 205-226.

Gallego Martínez, D., 2014. Agricultura, desarrollo económico y democracia.

Gkartzios, M., Norris, M., 2011. 'If You Build It, They Will Come': Governing property-led rural regeneration in Ireland. Land Use Policy 28, 486-494. https://doi. org/10.1016/j.landusepol.2010.10.002

Grimes, S., Precedo Ledo, A., 1990. Urban transition and local development in a peripheral region: the case of Galicia, Spain, in: Bannon, M.J., Bourne, L.S., Sinclair, S. (Eds.), Urbanisation and Urban Development - Recent Trends in a Global Context, IGU Commission on Urban Systems and Urban Development. University College Dublin, Dublin, pp. 97-107.

Lanero Táboas, D., 2016. La Revolución Verde en la España atlántica: la industria gandera (1955-1975), in: Old and New Worlds: Global Challenges of Rural History. Lisboa.

Loepfe, L., Martinez-Vilalta, J., Piñol, J., 2011. An integrative model of human-influenced fire regimes and landscape dynamics. Environmental Modelling \& Software 26, 1028-1040. https://doi.org/10.1016/j.envsoft.2011.02.015

López Iglesias, E., 2018. Desestruturación do medio rural e desorde do territorio: o pano de fondo do problema dos incendios forestais en Galicia. Coloquio Galaico-Portugués sobre incendios forestais. Unha nova xeraciónde lumes?, in: Coloquio Galaico-Portugués Sobre Incendios Forestais. Santiago de Compostela.

López Iglesias, E., 2000. O sector agrário galego ás portas do século XXI. Balance das súas transformacións recentes. Revista Galega de Economía 9, 167-196.

Marey Pérez, M.F., Bruña García, X., Picos Martín, J., Rodríguez Vicente, V., 2017. Eucalyptus \& Planificación forestal: El caso de Galicia, in: $7^{\circ}$ Congreso Forestal Español. Plasencia.

Martínez López, A., 2000. Perspectiva histórica de la ganadería gallega: de la complementariedad agraria a la crisis de la intensificación láctea (1850-1995), in: Fernández Prieto, L. (Ed.), Terra e Progreso: Historia Agraria da Galicia Contemporánea. Edicións Xerais de Galicia, Vigo, pp. 353-381.

Marull, J., Tello, E., Bagaria, G., Font, X., Cattaneo, C., Pino, J., 2018. Exploring the links between social metabolism and biodiversity distribution across landscape gradients: A regional-scale contribution to the land-sharing versus land-sparing debate. Science of The Total Environment 619-620, 1272-1285. https://doi.org/10.1016/j. scitotenv.2017.11.196

Murdoch, J., 2000. Networks - a new paradigm of rural development? Journal of Rural Studies 16, 407-419. https://doi.org/10.1016/S0743-0167(00)00022-X 
Niemeier, G., 1945. Tipos de población rural en Galicia. Estudios geográficos 6, 301-327.

Otero Rodríguez, T., 2018a. De la multifuncionalidad ganadera a la especialización láctea: la difusa transición pecuaria gallega (1920-1975), in: Transiciones en la agricultura y la sociedad rural. Los Desafíos Globales de La Historia Rural. II Congreso Internacional. Santiago de Compostela.

Otero Rodríguez, T., 2018b. Modernización en la agricultura gallega a través de la innovación pecuaria: la especialización láctea en Galicia (1920-1975), in: Transiciones en la agricultura y la sociedad rural. Los Desafíos Globales de La Historia Rural. II Congreso Internacional. Excma. Diputación Provincial da Coruña, Concello de Santiago, SEHA, Rural Report, Santiago de Compostela, p. 121.

Otero-Enríquez, R., Gómez Rodríguez, S., 2007. La desconcentración urbana en el sistema territorial de Galicia: un enfoque conceptual y empírico. Boletín de la Asociación de Geógrafos Españoles 259-277.

Padrón Castañeda, N., Barranco Reyes, J., 2014. Cambio climático e incendios de 5ª generación, in: Riesgos naturales y cambio climático. Colegio de Ingenieros de Montes, pp. 81-90.

Pérez-Alberti, A., Borobio-Sanchiz, M., Castillo-Rodríguez, F., Payán-Pérez, M., 2014. Metodología y clasificación de tipos de paisaje en Galicia. GOT, Revista de Geografia e Ordenamento do Território 259-282. https://doi.org/10.17127/got/2014.6.015

Precedo Ledo, A., Míguez Iglesias, A., Fernández Justo, M.I., 2008. Galicia: o tránsito cara a unha sociedade urbana no contexto da Unión Europea. Revista Galega de Economía 17, 89-108.

Rico Boquete, E., 2014. La industria del aserrío mecánico en Galicia, 1856-1935. Historia Agraria. Revista de agricultura e historia rural 83-116.

Schrijver, R., Poppe, K., Daheim, C., 2017. La agricultura de precisión y el futuro del sector agropecuario en Europa. Servicio de Estudios del Parlamento Europeo, Bruselas.

Silva-Pando, F.J., Pino-Pérez, R., 2016. Introduction of Eucalyptus into Europe. Australian Forestry 79, 283-291. https://doi.org/10.1080/00049158.2016.1242369

Soto Caba, M., 2013. Aproximación al origen y naturaleza de la conflictividad de las plantaciones de eucalipto (Eucalyptus ssp) en España, in: $6^{\circ}$ Congreso Forestal Español. Vitoria-Gasteiz.

Soto Fernández, D., 2016. De la multifuncionalidad a la especialización, la irrelevancia y la reinvención: los montes de Galicia durante la transición socio-ecológica (19602012), in: Old and New Worlds: The Global Challenges of Rural History. Lisboa.

Soto Fernández, D., González de Molina Navarro, M.L., Infante Amate, J., Guzmán, G., 2016. La evolución de la ganadería española (1752-2012). Del uso múltiple al uso alimentario. Una evaluación de la fiabilidad de los censos y de las estadísticas de producción, in: IV Seminario Anual de la SEHA. Madrid.

Stewart, S., Radeloff, V., Hammer, R., Hawbaker, T., 2007. Defining the Wildland Urban Interface. Journal of Forestry 105, 201-207. 
Tello, E., 2015. Transiciones socioecológicas en la agricultura: en busca del nexo oculto entre agroecología y biodiversidad., in: Los inciertos pasos desde aquí hasta allá: Altnernativas socioecológicas y transiciones poscapitalistas. Universidad de Granada, Granada, pp. 313-342.

Tello, E., Marull, J., Cattaneo, C., Padró, R., Pino, J., 2018. The loss of landscape ecological functionality in the Barcelona province (1956-2009): Can land-use legacies affect current biodiversity location?, in: Transiciones en la agricultura y la sociedad rural. Los desafíos globales de la Historia Rural. II Congreso Internacional. Excma. Diputación Provincial da Coruña, Concello de Santiago, SEHA, Rural Report, Santiago de Compostela, p. 73.

Vázquez González, I., Sineiro García, F., Lorenzana Fernández, R., 2008. Tipología de municipios rurales de Galicia por indicadores socioeconómicos, in: XII Congreso Internacional de Ingeniería de Proyectos (12th International Conference on Project Engineering). Zaragoza, pp. 1775-1786.

Veiras, X., Soto, M.Á., 2011. La conflictividad de las plantaciones de eucalipto en España (y Portugal). Greenpeace, Madrid.

Vicente Serrano, S.M., Lasanta Martínez, T., Cuadrat Prats, J.M., 2000. Influencia de la ganadería en la evolución del riesgo de incendio en función de la vegetación en un área de montaña: el ejemplo del valle de Borau (Pirineo aragonés). Geographicalia 33-57.

Viedma, O., Moity, N., Moreno, J.M., 2015. Changes in landscape fire-hazard during the second half of the 20th century: Agriculture abandonment and the changing role of driving factors. Agriculture, Ecosystems \& Environment 207, 126-140. https://doi. org/10.1016/j.agee.2015.04.011 